\title{
Ultrasonic modulation of persistent spectral holes in crystals
}

\author{
H. Lengfellner, a) T. R. Gosnell, R. W. Tkach, and A. J. Sievers \\ Laboratory of Atomic and Solid State Physics and Materials Science Center, Cornell University, Ithaca, New \\ York 14853
}

(Received 16 May 1983; accepted for publication 20 June 1983)

Persistent IR spectral holes in $\mathrm{KI}: \mathrm{ReO}_{4}^{-}$and in $\mathrm{RbI}: \mathrm{ReO}_{4}^{-}$crystals have been both modulated and erased with 10-MHz ultrasound. This mechanical modulation of the hole depth leads to a new phase-insensitive optical detector for ultrasonic waves in solids.

PACS numbers: 43.35.Sx, 78.20.Hp, 78.50.Ec, 43.35.Cg

A variety of inhomogeneously broadened optical and infrared transitions in solids display persistent spectral holes when irradiated with a narrowband laser. In the case of photochemical hole burning, absorption decreases at the laser frequency because the center undergoes an internal chemical change such as bond rearrangement or ionization. ${ }^{1-3}$ Nonphotochemical hole burning is thought to occur via photophysical rearrangement of the impurity and/or its neighbors. Persistent spectral holes have been observed for electronic transitions of photostable molecules imbedded in organic glasses ${ }^{4}$ and crystals ${ }^{5}$ and for vibrational transitions of photostable molecules in an organic glass ${ }^{6}$ and in alkali halides. ${ }^{7}$

We have investigated for the first time the influence of ultrasound on nonphotochemical persistent holes in alkali halides and find that ultrasonic waves modulate the hole depth. This mechanical modulation leads to a new optical method of monitoring ultrasound in crystals. In addition, prolonged exposure to ultrasonic radiation (in the absence of laser radiation) is found to permanently erase the persistent holes.

The experiments were performed on KI crystals doped with $0.05 \% \mathrm{KReO}_{4}$ and $2 \% \mathrm{NaI}$ and on $\mathrm{RbI}$ crystals doped with $0.4 \% \mathrm{RbReO}_{4}$, respectively. The crystals were mounted in the sample chamber of an optical cryostat and immersed in superfluid helium. Ultrasonic waves were generated and detected with a $10-\mathrm{MHz} X$-cut quartz transducer and commercial pulse-echo equipment.

Because of the large difference in thermal contraction between quartz and alkali halides, most elastic bonds between them tend to break at low temperatures. ${ }^{8}$ To counteract this problem, a special technique for bonding was developed. A drop of $n$-propyl alcohol was applied to the crystal surface and then a 20- $\mu$ indium foil was stretched over the surface forcing a thin film of fluid between the crystal surface and foil. The quartz-transducer was then bonded onto the indium foil with nonaq stopcock grease. This method prevented fast evaporation of the organic liquid and, because of the low freezing point of $n$-propyl alcohol, most of the thermal contraction had taken place before the bond solidified. With this bond, good echoes were obtained as the sample was cooled from room temperature to liquid helium temperature.

Holes were burned in the $\mathrm{RbI}$ crystal using the $\mathrm{CO}_{2}$ $10 \mathrm{P} 42$ line $(10.84 \mu)$ and in the KI sample with the $\mathrm{N}_{2} \mathrm{O} \mathrm{P} 19$

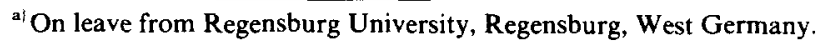

line $(10.47 \mu)$. In both cases the laser linewidth was less than $1 \mathrm{MHz}$. The transmitted laser radiation was detected with a $\mathrm{HgCdTe}$ detector. For measurements of the hole line shape, the intensity was strongly attenuated and a high sensitivity $\mathrm{Si}$ :As detector was used. Because of the large change in laser intensity during frequency scanning, the intensity was monitored with a reference detector as shown in Fig. 1 and the transmission signal was divided by this reference.

Hole modulation experiments were performed on a $\mathrm{KI}$ crystal immersed in superfluid helium. A $3-\mu$ s $10-\mathrm{MHz}$ ultrasound pulse was generated with the transducer and the resulting rectified transducer signal is shown in Fig. 2(a). The first large peak is due to pickup from the electrical excitation of the transducer. The following peaks are due to successive round trips of the ultrasonic wave packet.

In Fig. 2(b), the optical signal obtained from the $\mathrm{HgCdTe}$ detector is shown. After generation, the wave packet enters the illuminated volume and the first peak in the trace is obtained. Again, subsequent peaks identify repeated wave packet round trips but the optical signal also shows a continuous background increasing in strength during the first $100 \mu \mathrm{s}$. The insert shows the signal over an extended time scale. The spatial resolution of this detection scheme is limited only by the laser beam diameter, in this case $1 \mathrm{~mm}$.

The decreasing height of the transducer echoes shown in Fig. 2(a) is attributed to several effects. The amplitude loss due to absorption or energy leakage, for instance into the

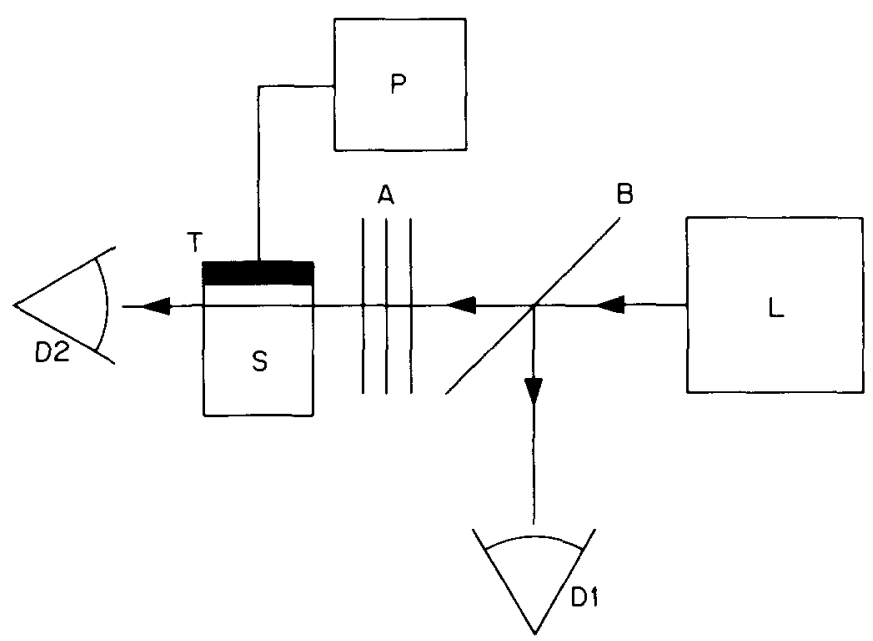

FIG. 1. Experimental configuration. $L=\mathrm{CO}_{2}$ or $\mathrm{N}_{2} \mathrm{O}$ laser, $D 1=$ pyroelectric reference detector, $D 2=\mathrm{HgCdTe}$ or $\mathrm{Si}$ :As photodetector, $A=$ attenuator, $S=$ sample, $T=$ transducer, $P=$ pulse generator and receiver. 


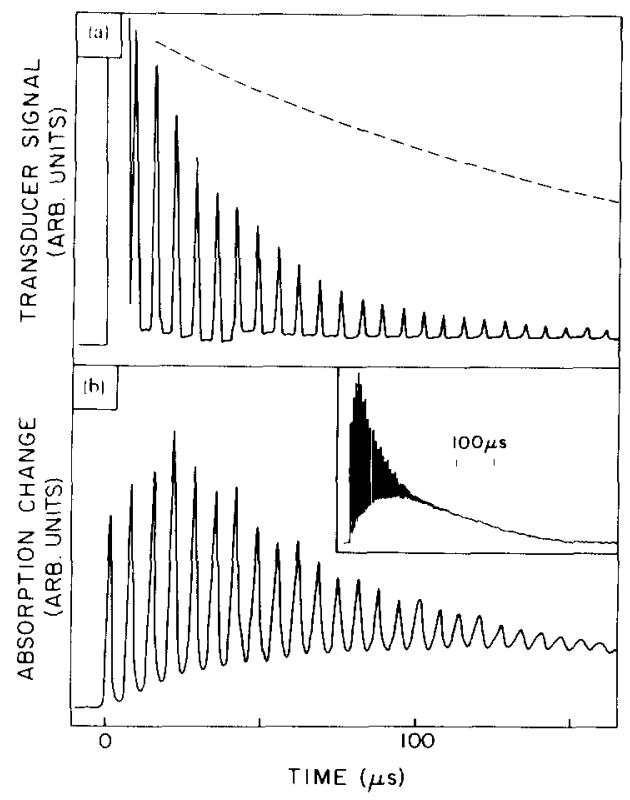

FIG. 2. Comparison of acoustic and optical signals vs time demonstrating modulation of persistent holes. (a) The rectified echo signal received at the transducer. (b) The modulated cw optical signal showing passage of the echoes through the beam as an increase in absorption. The inset shows the signal on an extended time scale. Sample $=\mathrm{KI}+0.05 \% \mathrm{KReO} 4+2 \% \mathrm{NaI}$, and the laser line $=\mathbf{N}_{2} \mathrm{O}$ P 19 .

helium bath, as estimated from the optical signal is indicated by the broken line in Fig. 2(a). The rapid decrease of echo signal height is partly due to diffraction. With increasing time, ultrasonic energy is lost from the wave packet and distributed over the crystal volume. This ultrasonic background is presumably quite random in phase at the transducer plane so that no electrical signal is produced. Additional transducer signal loss is due to path length differences. ${ }^{9}$

In contrast to the transducer signal, the optical signal shows both the background and the echoes. During the first $100 \mu \mathrm{s}$, the energy loss from the wave packet leads to an increase of the uniformly distributed intensity, which can be seen as an increase of the background signal in Fig. 2(b). After $200 \mu \mathrm{s}$, the coherent wave packet has lost its energy and the remaining trace shows the decay of the uniformly distributed ultrasound [insert of Fig. 2(b)]. The broken line in Fig. 2(a) is obtained by extrapolating this slow decay back to $t=0$. Our experimental results show that for this optical method, the phase of the ultrasonic waves can be of arbitrary value throughout the illuminated volume. The fact that the beam diameter was larger than the wavelength of the ultrasound supports this conclusion.

In order to investigate the influence of the ultrasound on the persistent holes we measured the hole line shape in a RbI crystal. After burning a hole, the laser intensity was attenuated to $1 \mu \mathrm{W} / \mathrm{cm}^{2}$. By slowly scanning the cavity length, the laser frequency was swept linearly in time over a range of $\pm 40 \mathrm{MHz}$. The hole shape was obtained from the ratio of the signal detector and the reference detector (see Fig. 1).

Figure 3(a) shows the hole shape immediately after hole burning. A similar scan made after the sample was irradiated

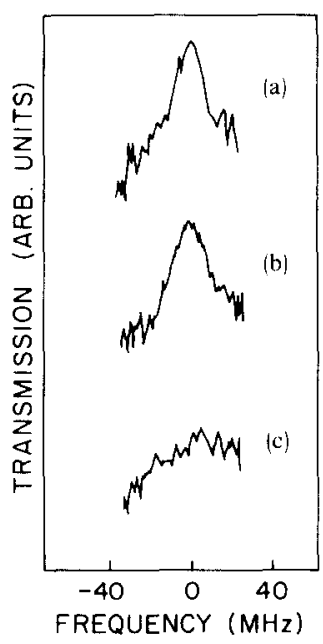

FIG. 3. Demonstration of the erasure of persistent holes by ultrasound. (a) Persistent hole spectrum immediately after burning. (b) Hole spectrum after two minutes laser dark time. (c) Hole spectrum two minutes after burning, but with ultrasound present during the laser dark time. Sample $=\mathrm{RbI}+0.4 \% \mathrm{RbReO}_{4}$, and the laser line $=\mathrm{CO}_{2} 10 \mathrm{P} 42$.

for two minutes with 10-MHz ultrasound is shown in Fig. 3 (c). For comparison two minutes of laser dark time (without ultrasound) elapsed between hole burning and scanning in Fig. 3(b). These experiments show that holes can be erased by ultrasonic waves. The erasing process is, however, not achieved immediately and depends on the intensity of the ultrasound. Our experiments thus indicate that holes are influenced in two ways by the ultrasound: There is a fast effect, faster than $1 \mu \mathrm{s}$, responsible for the echo traces, and a slow effect, leading to erasure of the hole.

The fast effect is a change in hole line shape due to the ultrasound. This has been confirmed by the following observation: During frequency scans we have applied ultrasound for short times. If the ultrasound is switched on at the hole maximum, the hole depth decreases by about $50 \%$. In the trace of Fig. 3(a), this would lead to a 50\% dip in the maximum of the curve. Off the hole center, the reduction of hole depth is smaller, and in the wings a small increase in hole depth was observed. This behavior suggests broadening of the hole by the ultrasound.

This broadening can be understood as frequency shifts of individual molecules induced by local ultrasonic strains. Since, at the hole's center frequency, the absorption can only increase due to these frequency shifts; the transmitted signal reflects the rectified displacement. This process is then completely independent of the phase of the ultrasound, provided that the acoustic wavelength is smaller than the beam diameter.

For comparison with other ultrasonic detectors, we have estimated the sensitivity of this new optical method. The only strain modes which interact with the excited state of the $v_{3}$ mode of $\mathrm{ReO}_{4}^{-}$are the long wavelength distortions of $A_{1 g}$ (spherical), $E_{g}$ (tetragonal and orthorhombic), and $T_{2 g}$ (trigonal) symmetry. Three coupling coefficients $A, B$, and $C$ then determine the dependence of the $v_{3}$ mode upon the lattice strains. Since uniaxial stress measurements have not been carried out on this system, we use the measured lattice constant dependence of the $v_{3}$ mode frequency for $\mathrm{ReO}_{4}^{-}$in alkali halides ${ }^{10}$ to estimate the hydrostatic coupling coefficient $A$.

To first order, the frequency shift $\Delta v$ and the strain $\Delta a$ / 
$a$ are related by the relation $\Delta v=-3 A(\Delta a / a)$, where $A=1.5 \times 10^{3} \mathrm{GHz} /$ unit strain for $\mathrm{KI}: \mathrm{ReO}_{4}^{-}$. A conservative estimate of the smallest strain detectable by observing the modulated laser intensity can be obtained by setting $\Delta v \approx \delta / 2$, where $\delta$ is the persistent hole linewidth. From this we obtain a minimum detectable strain on the order of $\Delta a$ / $a \sim 10^{-6}$. For longitudinal $10-\mathrm{MHz}$ ultrasonic waves with a wavelength $\lambda \sim 4 \times 10^{-2} \mathrm{~cm}$, a wave amplitude of $\sim 1 \AA$ should therefore be detectable.

Higher strain sensitivity should be possible with other defect systems. For example, the persistent photochemical hole ${ }^{1}$ of the $\mathrm{F}_{3}{ }^{+}$center in $\mathrm{NaF}$ has a homogeneous linewidth of $17 \mathrm{MHz}$ with a value $3 A=9.6 \times 10^{5} \mathrm{GHz} /$ unit strain. ${ }^{11}$ This system should have a minimum detectable strain $\Delta a /$ $a \sim 10^{-8}$, corresponding to an amplitude of $10^{-2} \AA$ for 10 $\mathrm{MHz}$ ultrasound, an amplitude which is comparable to those quoted for optical interferometric methods. ${ }^{9}$

In summary, we have demonstrated acoustic erasing and modulation of nonphotochemical persistent holes. The modulation experiments demonstrate a new phase insensitive optical method of high sensitivity for ultrasound detection in solids. This modulation has been explained as broadening of the hole line shape. Finally, we note that narrow photochemical peristent holes associated with electronic deffects should be particularly sensitive to ultrasonic modulation.

This work has been supported in part by the U.S. Army Research Office grant No. DAAG-29-83-K-0044 and by the National Science Foundation grant No. DMR-81-06097, Materials Science Center No. 5013. One of us (H.L.) acknowledges the receipt of a Fellowship from the Deutsche Forschungsgemeinschaft.

'R. M. Macfarlane and R. M. Shelby, Phys. Res. Lett. 42, 788 (1979); M. D Levensen, R. M. Macfarlane, and R. M. Shelby, Phys. Rev, B 22, 4915 (1980).

'B. M. Kharlamov, R. I. Personov, and L. A. Bykovskayn, Opt. Commun. 12, 191 (1974).

${ }^{3}$ A. A. Gorokhovskii, R. K. Kaarli, and L. A. Rebane, JETP Lett. 20, 216 (1974).

'J. M. Hayes, R. P. Stout, and G. J. Small, J. Chem. Phys. 74, 4266 (1981).

${ }^{5}$ F. G. Patterson, H. W. A. Lee, R. W. Olson, and M. D. Fayer, Chem Phys. Lett. 84, 59 (1981).

'M. Duk and H. H. Günthard, Chem. Phys. Lett. 64, 105 (1979).

'W. E. Moerner, A. J. Sievers, R.H. Silsbee, A. R. Chraplyvy, and D. K Lambert, Phys. Rev. Lett. 49, 398 (1982).

${ }^{8}$ A. Lehoczky, J. T. Lewis, and C. V. Briscoe, Cryogenics 6, 154 (1966)

${ }^{9}$ See for example, R. Truell, C. Elbaum, and B. B. Chick. Ultrasonic Methods in Solid State Physics (Academic, New York, 1969).

${ }^{10}$ W. E. Moerner, Ph.D. thesis, Cornell University, 1981.

${ }^{1} \mathrm{G}$. Baumann, F. Lanzl, W. von der Osten, and W. Waidelich, Z. Physik 197, $367(1966)$. 